\title{
Comparative Analysis of Traffic Light Control Mechanism for Emergency Vehicle
}

\author{
Jashvantkumar R. Dave *1, Shailesh Panchal *2
}

\begin{abstract}
Submitted: 26/08/2021 Accepted : 26/11/2021
Abstract: Traffic congestion during peak hours is a critical issue worldwide. Increasing number of vehicles, hence, traffic jams primarily near crossroads results into significant delay in emergency services vehicles. Surveys suggest that lives of many patients can be saved if emergency medical services could be reached in time. With the advancements in sensor and communication technology, it is now possible to provide improved solution to the delay resulting due to heavy traffic. Suggested solutions range from giving signaling priority to emergency vehicles to establishing a dedicated network to track the position of the emergency vehicle and ensuring an open route to the destination. This paper explores various mechanisms proposed for effective evacuation of vehicles on the route of emergency vehicle. Paper attempts to provide broader view and state of the work done in the said area. The study perceives the boundaries of existing traffic light pre-emption mechanisms, leading to several significant gaps that require further exploration. These gaps include implementation of proper system architecture, use of efficient communication technology, inclusion of real-time traffic data for optimized pre-emption of traffic light, prioritizing emergency vehicles to resolve conflict between multiple emergency vehicles and misuse prevention.
\end{abstract}

Keywords: Emergency vehicle routing, Intelligent Transport System, Traffic light control mechanism, VANET

This is an open access article under the CC BY-SA 4.0 license. (https://creativecommons.org/licenses/by-sa/4.0/)

\section{Introduction}

Demand of automobile has been increasing rapidly throughout the world. In India, a sale of passenger vehicle increased at the compound annual growth rate (CAGR) of $15.67 \%$ within the period of 2001 to 2010. In 2018, around 8.6 million more vehicles were manufactured as compared to vehicle manufactured in 2011[1]. Similar growth in vehicle production and sale is observed worldwide and thus majority of the countries are suffering from the issue of traffic congestion. Increased road traffic has multiple adverse effects as follows:

- Negative impact on environment: Emission of harmful gases in the environment has very serious effects on human health. In the UK, almost a third of total NOx was generated by road vehicles, with $23 \%$ from cars and light duty vehicles [2]. According to the report of World Health Organization (WHO)[3], around 4.2 million premature deaths have been reported in 2016 because of exposure to outdoor air pollution and vehicles travelling on road are one of the major source of outdoor air pollution.

- Loss of human life due to traffic accident: Road traffic accidents are increasing due to increase in vehicle population. WHO has launched the Global status report on road safety 2018 [4]. Report claims that the number of annual road traffic deaths has reached 1.35 million and road traffic injuries are 8th leading cause of death for people of all ages.

- Waste of time and money: In many parts of world, valuable time and money of the traveler is wasted because of traffic congestion issue. On average a commuter in Boston losing 149 hours a year due to congestion, costing \$2,205 per driver

\footnotetext{
${ }^{1}$ Gujarat Technological University, Ahmedabad-382424, India ORCID ID : 0000-0002-9881-5042

${ }^{2}$ Gujarat Technological University, Ahmedabad-382424, India ORCID ID : 0000-0003-4597-4063

* Corresponding Author Email: 179999913006@gtu.edu.in
}

in time loss [5]. In the UK, on average, Brits wasted 115 hours in congestion, costing the country $£ 6.9$ billion in 2019 [6].

- Increase in trip time for Emergency Vehicle: The sole purpose of emergency vehicle (EV) is to provide quick response to emergency situation. Lives and properties can be saved by providing timely service of EV. Many times delay in EV can cause severe catastrophe and the primary cause for EV delay is traffic congestion. In Ireland, around 700 fatalities are recorded every year due to delayed ambulance response [7].

Highest impact of increased road traffic can be observed at traffic intersections where more than one road intersects. To minimize the negative impact of increased traffic density, proper Traffic Light Control (TLC) mechanism needs to be implemented. This TLC mechanism ensures that all the vehicles travelling on road should get fair chance to cross the traffic intersection timely. In many countries, Most TLC mechanisms are statically programmed [8] i.e. they execute on predefined sequence and time duration. This type of TLC mechanism is only suitable for steady traffic flow. In reality, parameters such as time of the day (like peak hours), day of the week (like working day, non-working day) and climate conditions (summer / monsoon) can affect the traffic flow. To provide efficient traffic light schedule considering this frequently changing traffic pattern, TLC mechanism must adhere dynamic approach. In literature many such TLC mechanisms exist which considers real time traffic parameters and schedule traffic light accordingly. This paper focuses on different TLC mechanisms used for emergency vehicle clearance at traffic intersection. Generic TLC mechanism for EV clearance consists of three layers namely Physical layer, Communication layer and Decisionmaking layer. The schematic diagram of TLC mechanism is shown in Figure 1. Physical layer has Detectors, vehicles, EV and traffic light. The primary role of physical layer is to collect traffic parameters like density of vehicle, location and speed of vehicle, 
location, direction and speed of EV. Collected traffic parameters are then transferred to Decision making layer with the help of communication layer. Decision making layer consist of traffic light controller, analysed collected traffic parameters and based on that it will decide when to interrupt the current execution of traffic light and start green phase for traffic flow containing EV. The paper is organized as follows: In Section 2, the existing TLC mechanisms are presented by reviewing related literature. The features and effectiveness of the exiting TLC approaches are presented in Section 3. Section 4 contains simulation of different TLC mechanisms along with performance measurement based on various performance parameters. Significant gaps, which require further exploration, are presented in Section 5 and finally Section 6 concludes the paper.

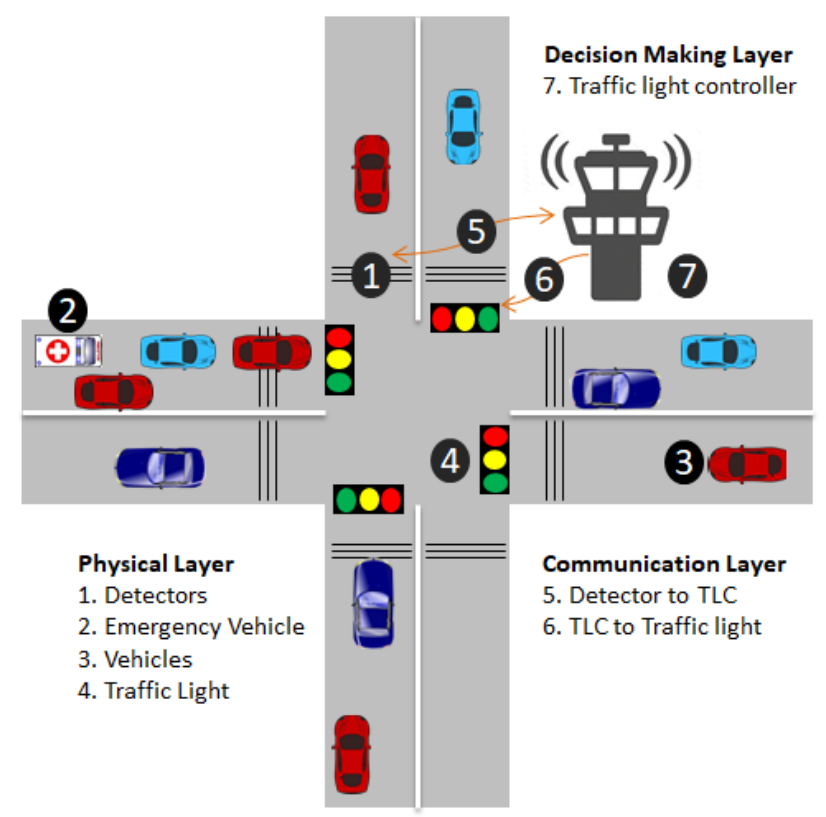

Fig. 1. Schematic diagram of TLC mechanism for EV clearance

\section{Related Work}

Efficient TLC mechanism is designed to provide hindrance free passage on the road so EV can move swiftly and safely. It aims to reduce the waiting time of $\mathrm{EV}$ at traffic intersections and to minimize negative impact of EV preemption on normal traffic. Some of the TLC mechanisms used for EV clearance introduced over the last few years are investigated in this paper.

Jianqi Liu et al. [9] proposed novel four-tier architecture for urbantraffic management using VANET, 5G wireless network, software-defined network (SDN), and mobile-edge computing (MEC) technologies. Real time traffic data is collected using Environment sensing layer, which is communicated to traffic light controller, and SDN enabled base station using VANET and 5G. Mobile edge computing layer is also implemented to minimize latency and quick response. For high-performance computing and to satisfy the storage requirement, cloud layer is added in the proposed architecture. Using this architecture, better communication and quick response can be achieved for timeconstrained applications.

To minimize queuing delay and to improve traffic fluency at traffic intersection, M. Bani Younes et al. [10] proposed Intelligent Traffic Light Controlling (ITLC) algorithm. Authors have introduced the concept of ready area which is decided based on average speed of all competing traffic flows and maximum allowed green time per traffic flow. Algorithm selects traffic flow with maximum traffic density within ready area and one nonconflicting traffic flow concurrently to cross traffic intersection so average trip time is reduced. Moreover, authors have also worked on scheduling of arterial traffic lights in which neighboring traffic lights will coordinated with each other to prepare efficient schedule for entire road network.

Shaaban et al. [11] proposed mechanism to reduce hindrance on the way of emergency vehicle by selecting an optimal route and applying preemption technique. In proposed mechanism, proper value of green time is calculated using signal switchover time, time required to discharge vehicles at intersection and fixed safety time. Decision to select early green or green extension is based on emergency vehicle's location and green time. Authors have also used Dijkastr's algorithm to calculate shortest path based on travel cost where travel cost is the function of length of road and congestion level at particular time interval. Proposed approach is simulated using microscopic simulator VISSIM and result shows that waiting time of emergency vehicle is very much reduced at the same time negative impact of preemption on general traffic is significantly reduced.

An approach to minimize average waiting time of emergency and non-emergency vehicle is presented by A. Khan et al. [12]. In the proposed approach, emergency vehicle broadcasts emergency priority request periodically. Once intersection controller receives this request, it calculates vehicle density using force resistive sensor on the approach from where emergency vehicle is travelling. Vehicle density, vehicle velocity, lane capacity, etc. are used to find effective green signal time and thus emergency vehicle's waiting time gets reduced. In the absence of emergency vehicle, among all four approaches, the approach with maximum vehicle density is selected to get green signal first and hence average waiting time for non-emergency vehicles is reduced.

Younes et al. [13] proposed dynamic and efficient traffic light scheduling algorithm to reduce the waiting time of emergency and non-emergency vehicle at signalized intersection. For reducing waiting time of non-emergency vehicles, traffic density for each traffic-flow is calculated using cooperative communication among travelling vehicles and highest-density competing traffic flow is scheduled to pass the signalized intersection first, coupled with another non-conflictive flow. All the emergency vehicles exist in the scenario reports its presence by broadcasting warning message using DSRC. Upon receiving warning message, algorithm checks that whether emergency vehicle is close to intersection or far from intersection. If it is close, traffic flow, containing emergency vehicle will be given highest priority and it will be scheduled first. To resolve conflict between more than one emergency vehicles approaching towards same intersection, type of emergency vehicle, distance from intersection and expected arrival time is considered.

To minimize average delay at traffic intersection, Pandit et al. [14] proposed two-phase mechanism using the concept of Oldest Job First (OJF) algorithm. During the first phase, control of traffic lights was formalized as a job-scheduling algorithm on processors, in which platoons represented jobs. The second phase demonstrates that how VANET can be used to group vehicles on the road into approximately equal size platoons. The OJF algorithm is applied for scheduling of these platoons and ensures reduction in waiting delay of the vehicles passing through intersection.

VANET based cooperative communication is used by Webar et al. [15] to model green wave for emergency vehicle. In proposed approach, authors have focused on solving the conflict between multiple emergency vehicles by assigning priority. To determine 
the value of free time (i.e. time required to clear the intersection), no. of waiting vehicles at intersection and time required by each waiting vehicle to clear intersection is used. Authors have compared the performance of proposed approach with two other approaches, which are currently in practice namely FAST and STREAM. The proposed approach outperforms when network of intersections is considered in the scenario.

Noori et al. [16] proposed a connected vehicle-based strategy to improve response time of emergency vehicle. In this strategy, realtime traffic density is calculated using IEEE 802.11p Vehicle to Infrastructure (V2I) communication, which is used to estimate the green time to clear the path for emergency vehicle at the traffic signal. Emergency vehicle broadcast beacon message periodically to notify its presence. Upon receiving beacon message, the centralized controller considers all traffic signals at emergency vehicle's route and changes the signal status to green before the EV reaches the traffic signal to minimize delay.

M. Asaduzzaman et al. [17] focused on Traffic Light Control mechanism using TSP (Transit Signal Priority) techniques (i.e. early green, green extension, phase rotation). This mechanism finds the value of lambda variable, which is the comparison between required time for early green and required time for green extension. It also checks the average waiting time for both the cases (early green and green extension) to make the decision. Multiple EV requests are solved by assigning priority weight based on emergency situation. Mechanism of Phase locking is mentioned, in which EV can lock appropriate phase for it. Locked phase cannot be booked by any other EV.

To understand the issues faced by emergency vehicle during the trip, video-based analysis of several emergency response trips is done by Buchenscheit et al. [18]. Authors have proposed an emergency vehicle warning system, which disseminates two different types of warning messages: One lightweight immediate warning message without route information in the single-hop distance from emergency vehicle and other full warning message containing route information using multi-hop mechanism. These messages assure that all the vehicles on the route of emergency vehicle must get warning sufficient in advance. Main focus of proposed mechanism is to get timely reaction of other drivers. Authors have also mentioned three different reactions of receiving vehicles, which have received warning message.

\section{Analysis of Existing Approaches}

In this section, some of the TLC mechanisms are analyzed based on the methodology and approach used for traffic light preemption. Mandatory features of traffic light preemption for EV clearance are also considered during analysis.

\subsection{Traffic Environment Sensing}

This mainly includes detection of EV and traffic density calculation. For designing efficient TLC systems for EV clearance, timely detection of EV and proper collection of real-time traffic data is first and foremost requirement. To fulfill these requirements, in literature various approaches are mentioned. We have categorized these approaches in two categories namely Sensor based sensing and Message based sensing. Some of the TLC mechanisms [9], [11], [12], [15] discussed here uses different on-road detectors like inductive loop, acoustic sensor, camera, force resistive sensor, magnetic sensor and ultrasonic sensor for detection of EV and few of these sensors are also used for traffic density calculations. However, the challenge lies with these sensors is its deployment and maintenance. Due to continuous movement of road traffic, replacement of these sensors is also troublesome. RFID and Infrared technology are also used by some researchers [19] but it suffers from the problem of limited coverage. Communication technologies like ZigBee and VANET [20] are used for message-based sensing. Among various communication technologies, VANET is the first choice of many researchers. Single-hop and multi-hop communication between Vehicle to Vehicle (V2V) and Vehicle to Infrastructure (V2I) eliminates the problem of limited coverage in VANET. When EV starts its trip, it starts broadcasting emergency message periodically. Using V2V and V2I communication, this emergency message can reach TLC in time and necessary steps can be initiated by TLC. VANET beaconing mechanism is used by vehicles to disseminate value of different vehicular parameters like average speed, travel direction, current location and destination. These beacon messages are used by TLC to generate real-time traffic information.

\subsection{Control Strategy for EV Preemption}

In literature, mainly static and dynamic strategies are available. In static strategy, traffic light sequence is pre-empted based on detection of EV at fixed distance (detection distance) from traffic light. In this strategy, deciding the value of detection distance is very crucial. Static strategy experiences poor performance when noticeable variation is observed in traffic density. In Dynamic strategy, traffic light controller collects real-time traffic data and based on that it decides optimal signal pre-emption time. TLC mechanism mentioned in [10], [11], [13], [15], [16] uses current traffic density and distance of EV from the traffic intersection for calculating green time required to clear the traffic. Moreover, approaches presented in [11], [16] consider start-up lost time and saturation headway along with traffic density and distance of EV to calculate required green time. Since dynamic strategy works on actual traffic data, it ensures better performance compared to static strategy.

\subsection{System Architecture}

In this paper, various TLC mechanisms are categorized in three groups namely Standalone architecture, Co-operative architecture and Centralized architecture. In [11]-[14], [17] authors have implemented TLC mechanism using the concept of standalone architecture. All the traffic light controllers are isolated from each other and operate separately at each traffic intersection. All these traffic light controllers extract information regarding real-time traffic and EV individually from its vicinity and clear traffic intersection one by one. One limitation of this architecture is traffic light pre-emption procedure cannot start until EV is detected locally. Some researchers [10], [15] have proposed TLC mechanisms for entire road network by adopting cooperative architecture. In this type of architecture all the traffic light controllers exchange the traffic data with neighboring traffic light controllers and thus they help each other to form green corridor on entire route of EV. Centralized architecture consists of central controller, which is connected to all the traffic light controllers exist on entire road network using different communication technologies. All these controllers forward traffic and EV information to central controller periodically. The central controller uses collected information to decide pre-emption schedule for all the traffic lights exist on the route of EV. In [9], [16] centralized architecture is used to minimize the effect of traffic congestion at intersections and to start traffic light preemption timely. 
Table 1. Analysis of TLC mechanisms for EV Clearance

\begin{tabular}{|c|c|c|c|c|c|}
\hline TLC Mechanism & $\begin{array}{l}\text { Traffic Environment } \\
\text { Sensing }\end{array}$ & Control strategy for EV preemption & System architecture & $\begin{array}{l}\text { Priority assignment } \\
\text { for EV }\end{array}$ & $\begin{array}{l}\text { Misuse } \\
\text { Prevention }\end{array}$ \\
\hline Jianqi Liu et al. [9] & $\begin{array}{l}\text { Inductive Loop, } \\
\text { VANET }\end{array}$ & Dynamic (Traffic Density) & $\begin{array}{l}\text { Layered } \\
\text { architecture with } \\
\text { Centralized control }\end{array}$ & - & - \\
\hline $\begin{array}{l}\text { M. Bani } \\
\text { Younes et al. [10] }\end{array}$ & VANET & $\begin{array}{l}\text { Dynamic (Traffic Density within Ready } \\
\text { Area, Time required to cross intersection, } \\
\text { Startup delay) }\end{array}$ & Co-operative & - & - \\
\hline K. Shaaban et al. [11] & Inductive Loop & $\begin{array}{l}\text { Dynamic (Traffic Density, Switching Time, } \\
\text { Safe Time Interval) }\end{array}$ & Standalone & - & - \\
\hline A. Khan et al. [12] & $\begin{array}{l}\text { Force Resistive } \\
\text { Sensor }\end{array}$ & $\begin{array}{l}\text { Dynamic (Distance of EV from intersection, } \\
\text { Speed of EV) }\end{array}$ & Standalone & - & - \\
\hline $\begin{array}{l}\text { M.B. } \\
\text { Younes et al. [13] }\end{array}$ & VANET & $\begin{array}{l}\text { Dynamic (Traffic Density, Expected time of } \\
\text { arrival) }\end{array}$ & Standalone & $\begin{array}{l}\text { EV Type, Distance } \\
\text { from Intersection, } \\
\text { Traffic density }\end{array}$ & - \\
\hline K. Pandit et al. [14] & VANET & $\begin{array}{l}\text { Dynamic (Expected arrival time of vehicle } \\
\text { platoon) }\end{array}$ & Standalone & - & - \\
\hline M. Weber et al. [15] & $\begin{array}{l}\text { Inductive Loop, } \\
\text { VANET }\end{array}$ & $\begin{array}{l}\text { Dynamic (No. of waiting vehicle, Time } \\
\text { required to cross intersection, Safety time) }\end{array}$ & Co-operative & EV Type & - \\
\hline Noori Hamed et al. [16] & VANET & $\begin{array}{l}\text { Dynamic (Traffic Density, Expected time of } \\
\text { arrival, Saturation Headway, Start-up lost } \\
\text { time) }\end{array}$ & Centralized & - & - \\
\hline $\begin{array}{l}\text { M. Asaduzzaman et al. } \\
\text { [17] }\end{array}$ & VANET & $\begin{array}{l}\text { Dynamic (Distance of EV from intersection, } \\
\text { Speed of EV) }\end{array}$ & Standalone & Emergency situation & - \\
\hline
\end{tabular}

\subsection{Priority Assignment for EV}

Most of the work reviewed here have considered ambulance, fire truck and police car in the category of EV but very few have discussed about the issue of resolving the conflict between more than one EV approaching towards same traffic intersection approximately at same time. In [15], conflict between EVs is resolved based on type of EV and its expected arrival time. TLC mechanism mentioned in [13] has presented EV priority as function of type of EV, distance from traffic intersection and current traffic density. Emergency scenario-based priority assignment is also proposed in [17].

\subsection{Misuse Prevention}

One of the basic requirements of TLC mechanism for EV preemption is that only authorized emergency vehicles can influence TLC mechanism. If no proper approach for misuse prevention is implemented, then it may possible that other vehicles could illegally send emergency vehicle warning messages to gain a driving advantage. In [18], [21], emergency vehicle warning messages are encrypted using public key encryption by Certificate Authority. IoT based authentication mechanism is proposed in [22], which take unique ID and photo of EV user and based on that generates exclusive (key, value) pair. If assigned (key, value) pair is not verified at hospital where EV is approaching within specified time period then EV user will be blocked.

Comparative analysis of all the reviewed TLC mechanisms is summarized in Table 1.

\section{Performance Evaluation}

\subsection{Experimental Setup}

In this section, performance of EVP-STC [12], WALABI [15] and
ETLSA [13] is evaluated on common simulation testbed. These three approaches are intelligent TLC mechanisms, which have implemented the strategy to minimize negative impact of traffic light pre-emption. Most preferred microscopic traffic simulator SUMO (Simulation of Urban MObility) [23] is used to generate traffic mobility scenarios for signalized road intersection. Using Python program, SUMO, TraCI interface and NS-2 we simulate the traffic in a city with intelligent traffic signals and change the signals status according to different traffic situations. On 132ft ring road, 9.5 KM long road segment between RTO circle and Shyamal crossroad of Ahmedabad is used for generating street network. Total five traffic intersections are considered, and all the intersections have four approaches contain two lanes in approaching and exiting directions. To study the effect of traffic density and EV detection distance on performance of TLC mechanism, different traffic scenarios are generated. The simulation parameters are summarized in Table 2.

Table 2. Simulation Parameters

\begin{tabular}{ll}
\hline Parameter & Value \\
\hline Length of road & $9.5 \mathrm{Km}$ \\
No. of Traffic intersection & 5 \\
Length of Road segment & $875 \mathrm{~m}-2560 \mathrm{~m}$ \\
Minimum Trip distance & $2375 \mathrm{~m}$ \\
Traffic density & $1000 \mathrm{veh} / \mathrm{hr}-6000 \mathrm{veh} / \mathrm{hr}$ \\
EV velocity & $16.66 \mathrm{~m} / \mathrm{s}$ \\
No. of lanes per approach & 2 \\
\hline
\end{tabular}

\subsection{Result Analysis}

During the investigation, our performance parameters are average waiting time of all traveling vehicles and emergency vehicle response time (EVRT). 


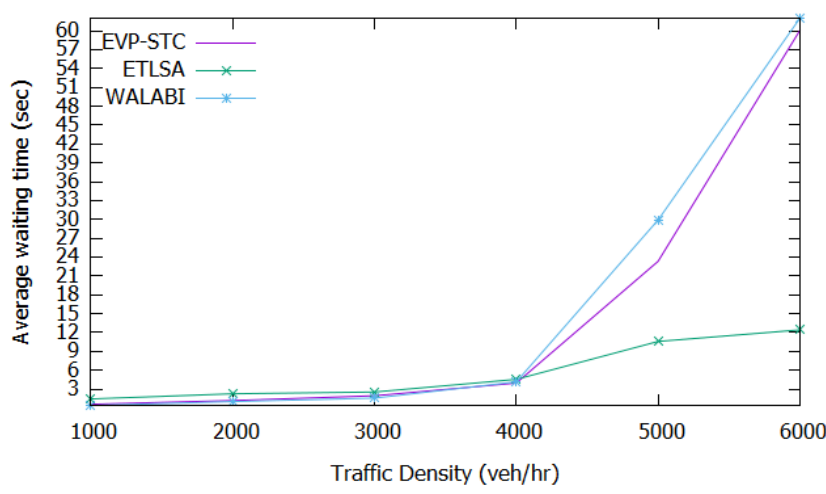

Fig. 2. Effect of traffic density on Average Waiting Time

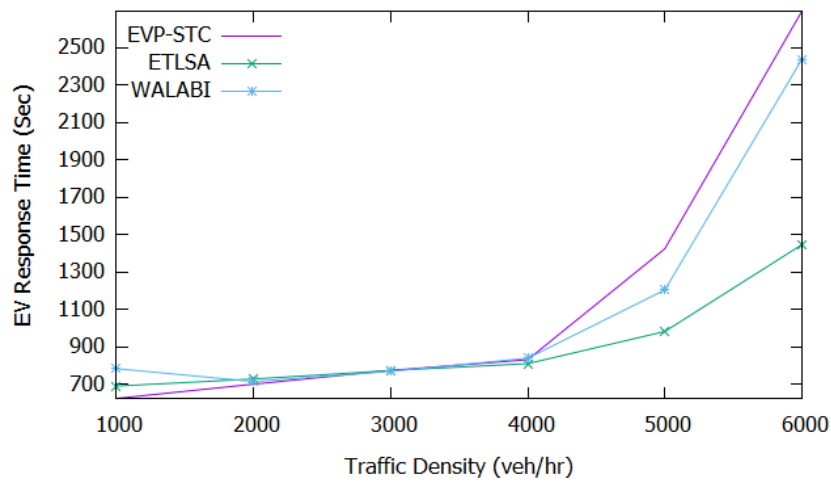

Fig. 3. Effect of traffic density on EV response time (EVRT)

\subsubsection{Effect of traffic density on performance parameters:}

Fig. 2 and Fig. 3 graphically demonstrate the effects of traffic density on average waiting time and EVRT respectively for all the three TLC mechanisms. Fig. 2 shows that for all the three TLC mechanisms, the average waiting time increases gradually with the increase in traffic density. Sudden rise is noticed once traffic density increases beyond the traffic saturation threshold for EVPSTC and WALABI whereas for ETLSA no major change is noticed. It is also observed that the average waiting time is reduced by $64 \%$ and $67 \%$ when implementing ETLSA, compared to the case when EVP-STC and WALABI is implemented respectively. This difference is due to the efficient design of ETLSA algorithm, in which eight different traffic flows are considered from which two most dense and non-conflicting traffic flows are given highest priority after EV and are opened in parallel, while in EVPSTC and WALABI the whole traffic is divided in four traffic flows only and each flow is signaled consecutively based on their priority.

It is also noticed that the average waiting time is little higher for low and medium traffic density with ETLSA as compared to EVPSTC and WALABI but for high traffic density, ETLSA outperforms EVP-STC and WALABI. This is because of the fact that ETLSA considers the distance of last vehicle from the traffic light to calculate the green phase time and for low and medium traffic, traffic density is low within green area but the distance of last vehicle from the traffic light within green area is more. So more green phase time is allocated to that edge and other vehicles on other edges needs to wait more.

From Fig. 3, we can observe that with increase in traffic density, EVRT has also increased. This is due to the hindrance caused because of increase in vehicles travelling on the path of EV. ELTSA outperforms EVP-STC and WALABI in terms of EVRT because in EVP-STC and WALABI, traffic density has direct implication on green phase time and hence increase in traffic density increases green phase time for dense traffic flow. More green-phase time for a specific traffic flow results into increase in waiting time of vehicle travelling on other traffic flows.

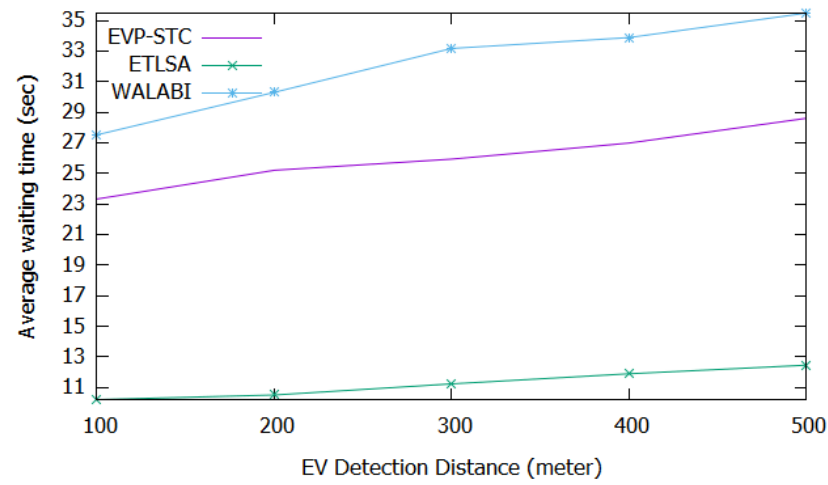

Fig. 4. Effect of EV detection distance on Average Waiting Time

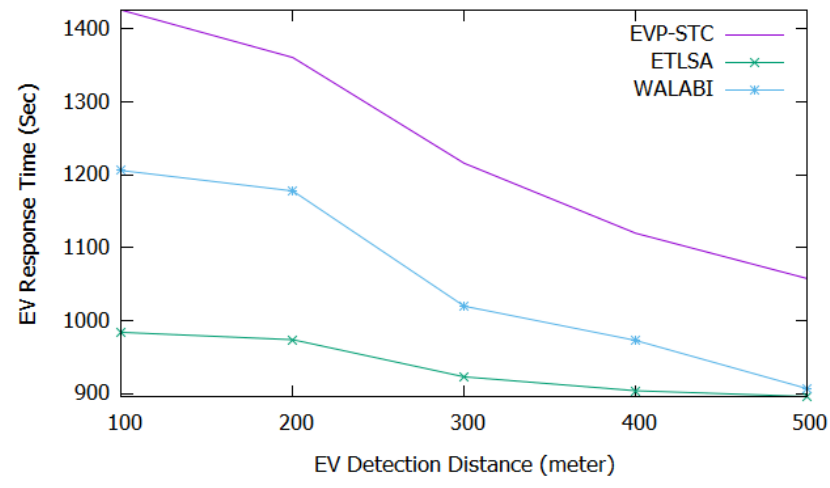

Fig. 5. Effect of traffic density on EV response time (EVRT)

\subsubsection{Effect of EV detection distance on performance parameters:}

Fig. 4 and Fig. 5 illustrate the effects of EV detection distance on average waiting time and EVRT respectively. By observing Fig. 4, we can clearly state that EV detection distance has less impact on average waiting time for all the TLC mechanisms tested here because little increase in average waiting time is observed upon increasing EV detection distance. This is due to the fact that all the mechanisms implemented here are deciding optimal value for traffic light preemption mainly based on the distance of EV from traffic light and speed of EV.

Fig. 5 illustrates that with increase in EV detection distance, EVRT decreases for all the TLC mechanisms investigated here. This is because of the fact that increases in $\mathrm{EV}$ detection distance, results in detection of EV sufficiently in advance hence TL preemption can be triggered at appropriate time. When comparing the performance of all the three TLC mechanisms investigated here, $15 \%$ improvement in EVRT is observed with WALABI compared to EVP-STC whereas, $11 \%$ improvement in EVRT is observed for ETLSA with compared to WALABI. This is justified by the fact that ELTSA algorithm, accurately calculates start time for traffic light preemption considering real-time traffic.

\section{Issues and Challenges}

This section hints about the sub-domains and scenarios, which can be explored further to improve the results achieved by existing TLC mechanisms.

- System architecture: Detecting EV at traffic light doesn't ensure optimal EVRT for highly dense traffic scenarios. To improve EVRT, EV should be detected sufficiently in 
advance so evacuation of all the vehicles between EV and traffic intersection can be done within proper time Centralized architecture-based TL preemption mechanisms can be explored.

- Communication technology: Among different communication technologies, VANET can be given more importance since it seamlessly supports V2X communication. Using VANET, collection and exchange of contro information could be done efficiently and hence the performance of TLC mechanism can be improved.

- Inclusion of real-time data: The value of various traffic parameters changes frequently and hence focus on designing dynamic strategy for TL preemption which uses real-time traffic parameters like traffic density, traffic speed, EV location and speed of EV, vehicle type to calculate optimal green phase time could result in significant improvement in EVRT.

- Misuse prevention: Lack of proper mechanism for misuse prevention might result in unauthorized vehicles gaining driving advantage. Inclusion of proper technique for validating EVs to ensure that only valid EVs can trigger the TL preemption can prevent the misuse.

- EV priority: Limited research has been done on handling simultaneous EV request because of which performance of EV trip time increases. Further studies can introduce efficient mechanism to assign priorities based on various criteria for resolving EV conflict.

\section{Conclusion}

Comparative analysis of different traffic light control mechanisms for emergency vehicle pre-emption is presented in this paper. Investigation includes approaches used for sensing traffic environment, strategies for traffic light pre-emption, architectures, conflict resolution between emergency vehicles and misuse prevention. Experimental results suggest that traffic density and early detection of emergency vehicle have higher impact on both average waiting time and emergency vehicle response time. More focus on inclusion of real-time traffic parameters and efficient communication technology like VANET for improvement in performance parameters is suggested. Inclusion of priority assignment to emergency vehicle and restricting unauthorized use of traffic light pre-emption can further improve result towards realistic scenario.

\section{References}

[1] S. Miglani, "The Growth of the Indian Automobile Industry: Analysis of the Roles of Government Policy and Other Enabling Factors." Springer Singapore, 2019, pp. 439-463 (2019).

[2] T. M. D Wakeling and NR Passant, "Uk informative inventory report.”, https://uk-air.defra.gov.uk/library/index

[3] "Effect of air pollution on human health.", https://www.who.int/news-room

[4] "The global status report on road safety 2018.", https://www.who.int/violenceinjury prevention/road safety status/2018/en/

[5] "Effect of traffic congestion in usa.", https://inrix.com/pressreleases/2019-traffic-scorecard-us/

[6] "Effect of traffic congestion in uk.", https://inrix.com/pressreleases/2019-traffic-scorecard-uk/

[7] S. Djahel, N. Smith, S. Wang, and J. Murphy, "Reducing emergency services response time in smart cities: An advanced adaptive and fuzzy approach,” in 2015 IEEE First International Smart Cities Conference (ISC2), pp. 1-8 (2015).

[8] V. Paruchuri, S. Chellappan, and R. B. Lenin, "Arrival time based traffic signal optimization for intelligent transportation systems," in
2013 IEEE 27th International Conference on Advanced Information Networking and Applications (AINA), pp. 703-709 (2013).

[9] J. Liu, J. Wan, D. Jia, B. Zeng, D. Li, C. Hsu, and H. Chen, "Highefficiency urban traffic management in context-aware computing and $5 \mathrm{~g}$ communication," IEEE Communications Magazine, vol. 55, no. 1, pp. 34-40, (2017).

[10] M. Bani Younes and A. Boukerche, "Intelligent traffic light controlling algorithms using vehicular networks," IEEE Transactions on Vehicular Technology, vol. 65, no. 8, pp. 58875899, (2016).

[11] K. Shaaban, M. A. Khan, R. Hamila, and M. Ghanim, "A strategy for emergency vehicle preemption and route selection," Arabian Journal for Science and Engineering, vol. 44, no. 10, pp. 8905-8913, (2019).

[12] A. Khan, F. Ullah, Z. Kaleem, S. Ur Rahman, H. Anwar, and Y. Cho, "Evp-stc: Emergency vehicle priority and self-organising traffic control at intersections using internet-of-things platform," IEEE Access, vol. 6, pp. 68 242-68 254, (2018).

[13] M. B. Younes and A. Boukerche, "An efficient dynamic traffic light scheduling algorithm considering emergency vehicles for intelligent transportation systems," Wireless Networks, vol. 24, no. 7, pp. 2451-2463, (2018)

[14] K. Pandit, D. Ghosal, H. M. Zhang, and C. Chuah, "Adaptive traffic signal control with vehicular ad hoc networks," IEEE Transactions on Vehicular Technology, vol. 62, no. 4, pp. 1459-1471, (2013).

[15] L. Bieker-Walz and M. Behrisch, "Modelling green waves for emergency vehicles using connected traffic data," in SUMO User Conference 2019, vol. 62, pp. 10-20, (2019).

[16] H. Noori, L. Fu, and S. Shiravi, "A connected vehicle based traffic signal control strategy for emergency vehicle preemption", Transportation Research Board 95th Annual Meeting, No. 16-6763, (2016).

[17] M. Asaduzzaman and K. Vidyasankar, "A priority algorithm to control the traffic signal for emergency vehicles," in 2017 IEEE 86th Vehicular Technology Conference (VTC-Fall), pp. 1-7 (2017).

[18] A. Buchenscheit, F. Schaub, F. Kargl, and M. Weber, "A vanetbased emergency vehicle warning system", pp. 1 - 8 (2009).

[19] A. Guerrero-Ib'ãnez, J. Contreras-Castillo, R. Buenrostro, A. B. Marti, and A. Reyes Mũnoz, "A policy-based multiagent management approach for intelligent traffic-light control," in 2010 IEEE Intelligent Vehicles Symposium, pp. 694-699, (2010).

[20] Y. Wang and F. Li, "Vehicular ad hoc networks," in Guide to wireless ad hoc networks, Springer, pp. 503-525, (2009).

[21] M. Masoud and S. Belkasim, "Wsn-evp: A novel special purpose protocol for emergency vehicle preemption systems," IEEE Transactions on Vehicular Technology, vol. 67, no. 4, pp. 36953700, (2017).

[22] S. Bhosale, N. Dhawas, and A. Burkul, "Vanet based communication for emergency vehicles," International Journal of Advanced Research in Computer Science and Electronics Engineering (IJARCSEE), vol. 2, no. 7, pp. 567-571, (2013).

[23] Vehicular traffic simulator. https://www.eclipse.org/sumo/ 